\title{
Dark Sky Collaborators: Arizona (AZ) Observatories, Communities, and Businesses
}

\author{
Elizabeth Alvarez del Castillo ${ }^{1}$, Christopher Corbally ${ }^{2}$, \\ Emilio E. Falco ${ }^{3}$, Richard F. Green ${ }^{4}$, Jeffrey C. Hall ${ }^{5}$ \\ and G. Grant Williams ${ }^{6}$ \\ ${ }^{1}$ AdC Consulting, Tucson, AZ, USA email: ema26@cornell.edu \\ ${ }^{2}$ Vatican Obs., Vatican City State email: corbally@as.arizona.edu \\ ${ }^{3}$ Smithsonian Astrophysical Obs, Cambridge, MA, USA email: falco@cfa.harvard.edu \\ ${ }^{4}$ Large Binocular Telescope Obs., Tucson, AZ, USA email: rgreen@lbto.org \\ ${ }^{5}$ Lowell Obs., Flagstaff, AZ, USA email: jch@lowell.edu \\ ${ }^{6}$ MMT Obs., Univ. of Arizona, Tucson, AZ, USA email: ggwilli@mmto.org
}

\begin{abstract}
With outdoor lighting ordinances in Arizona first in place around observatories in 1958 and 1972, then throughout the state since 1986. Arizonans have extensive experience working with communities and businesses to preserve our dark skies. Though communities are committed to the astronomy sector in our state, astronomers must collaborate with other stakeholders to implement solutions. Ongoing education and public outreach is necessary to enable ordinance updates as technology changes. Despite significant population increases, sky brightness measurements over the last 20 years show that ordinance updates are worth our efforts as we seek to maintain high quality skies around our observatories. Collaborations are being forged and actions taken to promote astronomy for the longer term in Arizona.
\end{abstract}

Keywords. Site Protection, Light Pollution, Lighting Ordinances, Education, Public Outreach

Given astronomys significant economic impact in the state, AZ supports protecting our resource of dark skies, but communities, astronomy, and businesses must collaborate and revisit the adequacy and enforcement of existing ordinances in a new effort to reduce light pollution, especially that which is associated with changes due to growth and new technologies. Ongoing education, relationships and collaboration are essential to success.

To implement solutions, astronomers collaborate with stakeholders. Statewide, relationships with stakeholders were critical when seeking to control a recently proposed proliferation of LED billboards. Northern AZ around Flagstaff has cultivated community pride in their dark skies, and astronomers continue to network and maintain relationships. A recent ordinance update in southern AZ around Tucson was almost torpedoed by a national interest group who arrived late in the process, but prior discussions, collaboration and compromise with local stakeholders saved the day.

The biggest challenge is anticipating future developments. Catching an issue while its young offers early opportunities to affect decisions; coming to the table late is complicated and may result in ill will. Astronomers must monitor lighting technologies and develop research-based recommendations to be prepared to address the effects of developing technology on our dark skies. Strong collaborations with stakeholders like the International Dark-Sky Association and Commission Internationale de l'Eclairage support these efforts.

To preserve our window to the universe: Designate a leader to cultivate community relationships. Remain vigilant, watching for developments. Communicate early and continue, as often as possible. Express concerns to the public and government entities. Maintain scientific ethics and standards; be credible. Collaborate and leave room for compromise. 